\title{
Quantifying simulator discrepancy in discrete-time dynamical simulators
}

\author{
Richard D. Wilkinson ${ }^{1,}{ }^{*}$, Michail Vrettas ${ }^{1}$, Dan Cornford ${ }^{2}$, and \\ Jeremy E. Oakley ${ }^{3}$ \\ ${ }^{1}$ School of Mathematical Sciences, University of Nottingham, \\ NG7 2RD, UK. Email: r.d.wilkinson@nottingham.ac.uk. \\ ${ }^{*}$ Corresponding author \\ ${ }^{2}$ School of Engineering and Applied Science, Aston University, \\ B4 $7 E T, U K$ \\ ${ }^{3}$ School of Mathematics and Statistics, University Of Sheffield, \\ S3 $7 R H, U K$
}

\begin{abstract}
When making predictions with complex simulators it can be important to quantify the various sources of uncertainty. Errors in the structural specification of the simulator, for example due to missing processes or incorrect mathematical specification, can be a major source of uncertainty, but are often ignored. We introduce a methodology for inferring the discrepancy between the simulator and the system in discrete-time dynamical simulators. We assume a structural form for the discrepancy function, and show how to infer the maximum likelihood parameter estimates using a particle filter embedded within a Monte Carlo expectation maximization (MCEM) algorithm. We illustrate the method on a conceptual rainfall runoff simulator (logSPM) used to model the Abercrombie catchment in Australia. We assess the simulator and discrepancy model on the basis of their predictive performance using proper scoring rules.
\end{abstract}




\section{Introduction}

The increasing usage of computer simulators in science and decision making raises many interesting statistical challenges. Because there is no natural variability in a simulator experiment, quantifying the degree of confidence in predictions is a task that needs to be explicitly undertaken by the modellers. For a given phenomenon and simulator of it, there are several sources of uncertainty: parametric uncertainty from not knowing the 'true' parameters values; initial condition uncertainty; uncertainty in measurements of the system (which is relevant if observations are used to improve forecast performance in a data assimilation scheme, or if forcing functions are imperfectly observed); numerical solver error; uncertainties induced by different temporal/spatial scales in the simulator and data; and finally, uncertainty from errors in the specification of the structural form of the simulator. Ideally, predictions should account for uncertainty, giving a forecast distribution that incorporates and combines uncertainty from all of these sources.

In this paper we focus on quantifying the simulator structural error in dynamical systems. There are a large variety of reasons why simulators are nearly always imperfect representations of the physical system they were designed to predict. For example, modellers' understanding of the system may be flawed, or perhaps not all physical processes were included in the analysis, and so on. This discrepancy has variously been called model error, model discrepancy, model structural error, and the term we use, simulator discrepancy. Once we accept the existence of simulator discrepancy, it is natural to ask whether we can either improve the simulator or quantify the error. 
The modeller might seek to improve their simulator through more accurate theory. Instead, we ask what can be learnt empirically about the simulator discrepancy, using past predictions and subsequent system observations.

While many methods have been proposed for dealing with parametric and initial condition uncertainty (Saltelli et al., 2000; Oakley and O'Hagan, 2002) and controlling numerical errors (Oberkampf and Trucano, 2008), methodology for quantifying simulator discrepancy is less well developed. The methods that have been proposed broadly classify into subjective methods that rely on expert knowledge (Goldstein and Rougier, 2009; Vernon et al., 2010; Strong et al., 2011), metric based methods to quantify the degree of error in past performance (Beven, 2006), turning deterministic dynamics into stochastic dynamics (see for example, Crucifix and Rougier (2009)), allowing parameters to vary through time (Kuczera et al., 2006; Reichert and Mieleitner, 2009), using ensembles of predictions from different simulators (Smith et al., 2009; House et al., 2011), data assimilation based methods (Griffith and Nichols, 2000), and direct statistical modeling of the simulator discrepancy (Kennedy and O'Hagan, 2001; Higdon et al., 2008; Goldstein and Rougier, 2009).

The method that is developed here is most closely related to the methodology proposed in Kennedy and O'Hagan (2001). They modelled the simulator discrepancy as a state dependent random function using a Gaussian process model. Their approach was for a static experimental situation in which observations were made for different values of the input conditions. The approach does not easily extend to the analysis of dynamical systems. To see why, suppose the output of the simulator is the prediction of a time-series 
of observations, $y_{1}, \ldots, y_{n}$. Under the approach in Kennedy and O'Hagan (2001), the discrepancy would be a function from the initial conditions to a time-series of length $n$. When $n$ is moderate to large in size, unless a suitably large number of independent trials (time-series) are available then we are unlikely to be able to successfully model the discrepancy. By considering the simulator discrepancy on the level of the dynamics, rather than the static form used in Kennedy and O'Hagan (2001), we reduce the dimension of the input and output space of the discrepancy function. Their approach is also not suitable in situations where we want to combine the simulator predictions with past observations in a data assimilation scheme in order to improve performance, which is common in many fields.

In this paper we focus solely on dynamical systems, where we assume there is a state variable $\mathbf{x}$ evolving through time which is noisily observed at discrete times, giving equally spaced observations $\mathbf{y}_{1}, \ldots, \mathbf{y}_{T}$. We aim to quantify errors in the prescribed dynamics of the simulator, and to learn the simulator discrepancy as a function of the current state vector. Quantifying the simulator discrepancy can be thought of as involving two separate issues: estimating the direction and magnitude of the bias; and quantifying the remaining uncertainty. We aim to do both, modelling the bias using a simple linear regression and quantifying the remaining uncertainty using an additive Gaussian white noise term. Although this is a simple model for the discrepancy, it should be contrasted with the usual approach in data assimilation schemes, which is to either ignore simulator discrepancy, or to use just an additive Gaussian white noise term.

Learning the discrepancy on the dynamics is inferentially difficult, as the 
true state $\mathbf{x}_{t}$ is never observed. The simulator dynamics are a map from the state vector $\mathbf{x}$, to another state at a later time, and it is here where we seek to train the discrepancy, but using only noisy observations $\mathbf{y}_{1}, \ldots, \mathbf{y}_{T}$.

The focus of our approach is on improving the predictive power of the simulator. We aim to give probabilistic predictions of future observations that adequately represent the uncertainty in our predictions. Given observations up to time $t, \mathbf{y}_{1}, \ldots, \mathbf{y}_{t}$, we aim to provide forecasts $\pi\left(\mathbf{y}_{t+k} \mid \mathbf{y}_{1}, \ldots, \mathbf{y}_{t}\right)$ of future events so that the future holds fewer surprises, in the sense that the tails of our distribution are neither too light nor too heavy. This approach is in contrast to focussing on the explanatory power of the simulator, where we would instead aim to achieving a good fit of the simulations to previously observed data (Shmueli, 2011). We do not address the issue of calibrating unknown simulator parameters here, but instead assume that we are provided with a precalibrated simulator in order to quantify its prediction error.

The structure of the paper is as follows. In the next section we describe the framework used to quantify the discrepancy, the methodology to learn the discrepancy, and comment on how to assess probabilistic forecasts made by dynamical systems using scoring rules. In Section 3 we illustrate the methodology on a conceptual rainfall-runoff simulator of the Abercrombie water-basin in Australia that has been the focus of several previous uncertainty quantification studies in hydrology. Section 4 offers discussion. A further case study and technical details of the algorithm are available in the online supplementary material. 


\section{Theory}

\subsection{Statistical forecasting framework}

We consider simulators of dynamical systems in which a state vector evolves in time and is noisily observed at regular intervals. Let $\mathbf{x}_{t} \in \mathbb{R}^{d}$ denote the value of the state vector at time $t$, and let $\mathbf{x}_{0: T}=\left\{\mathbf{x}_{0}, \ldots, \mathbf{x}_{T}\right\}$. We assume we are given an imperfect simulator of the system dynamics, $\mathbf{f}$, that is used to predict one time-step ahead

$$
\mathbf{x}_{t+1}=\mathbf{f}\left(\mathbf{x}_{t}, \mathbf{u}_{t}\right)
$$

For example, $\mathbf{f}$ could be a simulator that numerically integrates a system of differential equations $\frac{\mathrm{dx}}{\mathrm{dt}}=\mathbf{h}(\mathbf{x}, \mathbf{u}, t)$ with $\mathbf{x}_{t}$ as the initial condition. The vector $\mathbf{u}_{t}$ contains the forcing functions required by the simulator for the time period in question, and is included in the notation to emphasise that the simulator is a fixed function, not varying through time. Note that we assume the simulator has been calibrated previously, so that there are no unknown simulator parameters that need to be estimated.

We now impose a statistical framework that allows us to relate the simulator to the observations. This consists of two parts; the first relates the observations to the system (the measurement process), and the second relates the simulator prediction to the system (the simulator discrepancy). Let $\mathbf{y}_{0: T}=\left\{\mathbf{y}_{0}, \ldots, \mathbf{y}_{T}\right\}$ denote a sequence of observations of the state that are conditionally independent given $\mathbf{x}_{1}, \ldots, \mathbf{x}_{T}$ and assume that $\mathbf{y}_{t}=g\left(\mathbf{x}_{t}\right) \in \mathbb{R}^{p}$, where $g(\cdot)$ is a stochastic mapping, and that the observation likelihood, 
$\pi\left(\mathbf{y}_{t} \mid \mathbf{x}_{t}\right)$, is known and can be evaluated point-wise.

The second part of the statistical framework is to relate the simulator to reality, by specifying a model of the simulator discrepancy. A common approach in data assimilation is to model the discrepancy as a white noise term, so that errors are independent and identically distributed. This is equivalent to making the assumption that the prediction error of $\mathbf{f}$ is similar in all parts of space. However, in many scenarios the simulator discrepancy is smaller in some regions of space and larger in others. This occurs in the free-fall case study in the supplementary material where we consider a simulator of a falling object with the wrong specification of air-resistance. At low velocities the simulator is accurate, but at higher velocities the simulator error is large. Representing simulator discrepancy as a white noise process ignores this subtlety.

To account for varying simulator accuracy in different parts of space, we introduce a state-dependent simulator discrepancy $\boldsymbol{\delta}(\cdot)$, which is a function of the current state and forcings. We assume that the system dynamics are

$$
\mathbf{x}_{t+1}=\mathbf{f}\left(\mathbf{x}_{t}, \mathbf{u}_{t}\right)+\boldsymbol{\delta}\left(\mathbf{x}_{t}, \mathbf{u}_{t}\right)
$$

Contrast these dynamics with the simulator dynamics in Equation (1). The aim of this paper is to describe methodology to infer the functional form of $\boldsymbol{\delta}$, and to show that the effort of moving from a white simulator discrepancy to a state-dependent discrepancy can significantly improve the performance of the forecasting system. We assume a simple parametric form for $\boldsymbol{\delta}$ linear in the parameters and use ordinary least squares regression to estimate the 
unknown parameters in $\boldsymbol{\delta}$.

Let $\boldsymbol{\delta}(\mathbf{x}, \mathbf{u})=\left(\delta_{1}(\mathbf{x}, \mathbf{u}), \ldots, \delta_{d}(\mathbf{x}, \mathbf{u})\right)^{\top}$. For ease of exposition, we assume that $\delta_{i}(\mathbf{x}, \mathbf{u})$ and $\delta_{j}(\mathbf{x}, \mathbf{u})$ are conditionally independent given $\mathbf{x}$ (they are unconditionally dependent), so that we we can consider the simulator discrepancy in each of the $d$ dimensions of $\mathbf{x}$ separately. We assume that

$$
\delta_{j}(\mathbf{x})=\mathbf{p}_{j}(\mathbf{x}, \mathbf{u}) \boldsymbol{\beta}_{j}+\epsilon_{j}
$$

where $\boldsymbol{\beta}_{j}$ is a vector of $J$ unknown parameters, $\mathbf{p}_{j}=\left(p_{j}^{(1)}, \ldots, p_{j}^{(J)}\right)^{\top}$ is a row vector of $J$ specified functions of $\mathbf{x}$ and $\mathbf{u}$, and $\epsilon_{j} \sim \mathcal{N}\left(0, \tau_{j}\right)$ independently sampled at every occurrence. Let $\boldsymbol{\theta}$ denote the collection of the $d \times(J+1)$ unknown parameters in $\boldsymbol{\delta}$. For a deterministic simulator, the probability density function for the system dynamics of $\mathbf{x}$ assumed by our statistical framework is

$$
\pi\left(\mathbf{x}_{t+1} \mid \mathbf{x}_{t}, \boldsymbol{\theta}\right)=\prod_{j=1}^{d} \frac{1}{\sqrt{2 \pi \tau_{j}}} \exp \left[-\frac{1}{2 \tau_{j}}\left(x_{j, t+1}-f_{j}\left(\mathbf{x}_{t}, \mathbf{u}_{t}\right)-\mathbf{p}_{j}\left(\mathbf{x}_{t}, \mathbf{u}_{t}\right) \boldsymbol{\beta}_{j}\right)^{2}\right]
$$

where $f_{j}$ is the $\mathrm{j}^{\text {th }}$ dimension of the simulator output, and $x_{j, t+1}$ is the $\mathrm{j}^{\text {th }}$ component of $\mathbf{x}_{t+1}$. We do not explicitly include the forcings $\mathbf{u}$ in the density notation, as we assume they are observed without error.

Estimation of the simulator discrepancy for the dynamics of $\mathbf{x}$, can raise philosophical difficulties. Unobservable quantities can be problematic as they are in some sense merely labels; it can be unclear what, if any, physical reality they represent. In conceptual models, $\mathbf{x}$ is often viewed only as a useful tool for modelling and forecasting purposes, but not necessarily as having an 
operationally defined physical meaning. We can avoid the problem of talking of the error in the dynamics of (the label) $\mathbf{x}$ by thinking of $\boldsymbol{\delta}$ as a way of decreasing/quantifying errors in forecasts of the observables $\mathbf{y}$, and choosing not to focus on a direct interpretation of $\boldsymbol{\delta}$. In the next section, we introduce methodology for estimating $\boldsymbol{\theta}$. We drop the use of bold notation for vector quantities.

\subsection{Inference for $\delta(\cdot)$}

Inferring the shape of the simulator discrepancy is difficult, as it acts on the dynamics of the unobserved state vector, and thus the likelihood function $L(\theta)=\pi\left(y_{1: T} \mid \theta\right)$ is unknown in closed form ffor all nonlinear simulators. By introducing the hidden state trajectory $x_{0: T}$ into the calculation, the conditional independence structure of the statistical framework can be used to gain a degree of tractability. The likelihood of $\theta$ given $x_{0: T}$ and $y_{0: T}$ is

$$
\pi\left(x_{0: T}, y_{0: T} \mid \theta\right)=\left(\prod_{t=0}^{T} \pi\left(y_{t} \mid x_{t}\right)\right)\left(\prod_{t=0}^{T-1} \pi\left(x_{t+1} \mid x_{t}, \theta\right)\right) \pi\left(x_{0}\right)
$$

allowing the EM algorithm (Dempster et al., 1977) to be used to find the

maximum likelihood estimate, $\hat{\theta}=\arg \max _{\theta} L(\theta)$, by using $x_{0: T}$ as the missing data. The EM algorithm is iterative, generating a sequence $\theta^{(1)}, \theta^{(2)}, \ldots$ with $\theta^{(n+1)}=\arg \max _{\theta} Q\left(\theta, \theta^{(n)}\right)$ where

$$
Q\left(\theta, \theta^{(n)}\right)=\mathbb{E}_{X}\left[\log \pi\left(X_{0: T}, y_{0: T} \mid \theta\right) \mid y_{0: T}, \theta^{(n)}\right]
$$


so that $\theta^{(n)}$ converges to a maxima of $L(\theta)$. The expectation in Equation (6) is taken with respect to the smoothing distribution $\pi\left(x_{0: T} \mid y_{0: T}, \theta^{(n)}\right)$, which is unknown in general and cannot be computed analytically. However, we can sample from $\pi\left(x_{0: T} \mid y_{0: T}, \theta^{(n)}\right)$ using sequential Monte Carlo methods. If $\left\{x_{0: T}^{(i)}\right\}_{i=1, \ldots, M}$ are samples from $\pi\left(x_{0: T} \mid y_{0: T}, \theta^{(n)}\right)$, we can approximate $Q\left(\theta, \theta^{(n)}\right)$ by

$$
\tilde{Q}\left(\theta, \theta^{(n)}\right)=\frac{1}{M} \sum_{i=1}^{M} \log \pi\left(x_{0: T}^{(i)}, y_{0: T} \mid \theta\right)
$$

and then seek to maximize $\tilde{Q}$, allowing us to bypass the computationally intractable expectation. A consequence of using the Monte Carlo EM algorithm, is that we lose the likelihood-ascent property of the standard EM algorithm, and so cannot guarantee convergence (Wei and Tanner, 1990). However, the number of Monte Carlo samples, $M$, can be increased for each iteration of the EM algorithm, so that the Monte Carlo error in the estimation of the expectation decreases as we converge on the maximum likelihood estimate $\hat{\theta}$ (Caffo et al., 2005).

Substituting Equation (5) into Equation (7) reduces the problem to maximising

$$
\sum_{i=1}^{M} \sum_{t=0}^{T-1} \log \pi\left(x_{t+1}^{(i)} \mid x_{t}^{(i)}, \theta\right)
$$

with respect to $\theta$, where we have used the assumption that the prior distribution for $x_{0}$ and the observation process do not depend on $\theta$. For various choices of parametric family for $\delta$, Equation (8) can be maximized analytically. In particular, if $\delta$ is a linear model with Gaussian noise, such as in Equation (3), then when we substitute Equation (4) for $\pi\left(x_{t+1} \mid x_{t}, \theta\right)$, and recall that we are assuming conditional independence between the components 
of $\delta$, the maximization problem in Equation (8) separates into $d$ minimization problems: for $j=1, \ldots, d$ minimize

$$
\frac{1}{2 \tau_{j}} \sum_{i=1}^{M} \sum_{t=0}^{T-1}\left(x_{j, t+1}^{(i)}-f_{j}\left(x_{t}^{(i)}, u_{t}\right)-p_{j}\left(x_{t}^{(i)}, u_{t}\right) \beta_{j}\right)^{2}+\frac{1}{2} M T \log \tau_{j} .
$$

These optimization problems can be seen to be equivalent to the classical least squares optimization. Let $v_{j}$ be the response vector for optimization $j$, found by stacking elements $x_{j, t+1}^{(i)}-f_{j}\left(x_{t}^{(i)}, u_{t}\right)$ for $i=1, \ldots, M$ and $t=0, \ldots, T-1$, and let $Z_{j}$ denote the corresponding design matrix found by stacking the rows $p_{j}\left(x_{t}^{(i)}, u_{t}\right)$ in the same order as for $v_{j}$. Maximizing Equation (9) then gives

$$
\begin{aligned}
\hat{\beta}_{j} & =\left(Z_{j}^{\top} Z_{j}\right)^{-1} Z_{j}^{\top} v_{j} \\
\hat{\tau}_{j} & =\frac{1}{M T}\left(v_{j}-Z_{j} \hat{\beta}_{j}\right)^{\top}\left(v_{j}-Z_{j} \hat{\beta}_{j}\right),
\end{aligned}
$$

which are the usual maximum-likelihood estimates.

To generate sample trajectories, $x_{0: T}$, from $\pi\left(x_{0: T} \mid y_{0: T}, \theta\right)$, we use the bootstrap particle filter (Gordon et al., 1993; Doucet et al., 2001) and approximate the filtering distributions by a sample of $N$ weighted particles. Details of the algorithm are given in the supplementary material. While in theory the filter generates $N$ smoothed trajectories, in practice the marginal distribution of $x_{0}$ will be degenerate, with typically the same value of $x_{0}$ being observed in all $N$ trajectories. To generate $M$ smoothed trajectories, we implement $M$ independent filters, and randomly pick a single smoothed trajectory from the final filtering distribution in each filter. Because each filter is independent, we avoid the problem of degeneracy for $x$ values towards the 
start of the time-series. An alternative way to avoid degeneracy would be to use a particle smoother, such as that suggested by Godsill et al. (2004), but at the cost of making parallelization more difficult.

Because we are using the MCEM algorithm with finite sample size, the parameter estimates will continue to fluctuate even after having essentially converged. A stopping rule can be used to decide when to terminate the iterations in the EM algorithm, such as requiring a maximum percentage change in the MLE estimates over consecutive iterations. The stringency of the stopping criterion applied will depend on the size of $N$ and $M$ and on the identifiability of the discrepancy parameters.

A drawback of using the EM algorithm to estimate the MLEs is that error estimation is difficult as the marginal likelihood is not directly available. Standard error estimates are usually found by estimating the Hessian matrix using numerical differentiation, which can then be inverted to estimate the asymptotic variance of the MLE. For example, the supplemented EM algorithm (Meng and Rubin, 1991) uses an identity relating the Hessian matrix to the second derivative of $Q$ and the first derivative of the EM operator (i.e., the derivative of $\left.M\left(\theta^{(n)}\right)=\arg \max _{\theta} Q\left(\theta, \theta^{(n)}\right)\right)$. These approaches are unlikely to work for the MCEM algorithm. Because we approximate $Q$ by a Monte Carlo sum in the MCEM algorithm, numerical differentiation of $Q$ and of $M\left(\theta^{(n)}\right)$ is likely to be both prohibitively expensive (computationally) and unstable in most cases. As the focus of our paper is on improving the predictive power of simulators, rather than on the value of the estimated discrepancy, we do not focus on the uncertainty of the parameter estimates here. If uncertainty estimates of the parameters are required, then a Markov 
chain Monte Carlo (MCMC) approach is likely to be a simpler way to access the uncertainty distributions than the EM algorithm, although this will require considerably more computation.

\subsection{Assessing forecasting systems}

Our motivation for quantifying simulator error is to improve forecasting power, both in terms of reducing absolute error and quantifying uncertainty. As the majority of statistical diagnostic tools are designed to assess explanatory power rather than predictive power (Shmueli, 2011), we now make clear how we will judge the success or otherwise of a forecast.

We base the assessment on the ability to predict future observations given past observations, via the use of the k-step-ahead forecast distributions $\pi\left(y_{t+k} \mid y_{1: t}\right)$. We use a training sequence of data $y_{1: T_{1}}^{(1)}$ to train the model, and then use an independent validation data set $y_{1: T_{2}}^{(2)}$ in the testing. To find $\pi\left(y_{t+k} \mid y_{1: t}\right)$ we use a data assimilation scheme to obtain the filtering distributions $\pi\left(x_{t} \mid y_{1: t}\right)$, before propagating these through Equation (2) to find $\pi\left(x_{t+k} \mid y_{1: t}\right)$ and then through the observation process to find $\pi\left(y_{t+k} \mid y_{1: t}\right)$. It is not possible to analytically calculate these distributions and so all calculations are done using weighted ensembles of particles obtained from the particle filter.

We wish to assess both the bias and the uncertainty quantification of the forecasts. To assess the bias, we only need the means of the forecasts. Let $m_{t}(k)=\mathbb{E}\left(y_{t+k} \mid y_{1: t}\right)$ be the mean $k$-step-ahead forecast at time $t$. We use the mean-square-error (MSE) and the Nash-Sutcliffe (NS) statistic (Nash 
and Sutcliffe (1970)) applied to the mean forecast

$$
\operatorname{MSE}=\frac{1}{T-k} \sum_{t=1}^{T-k}\left(y_{t+k}-m_{t}(k)\right)^{2}, \quad \mathrm{NS}=1-\frac{\sum_{t=1}^{T-k}\left(y_{t+k}-m_{t}(k)\right)^{2}}{\sum_{t=k+1}^{T}\left(y_{t}-\bar{y}\right)^{2}}
$$

to assess the accuracy of the mean forecast. The Nash-Sutcliffe statistic is an analogue of the coefficient of determination, $R^{2}$, and is commonly used in hydrology to assess simulator accuracy. It compares the mean forecast performance with the performance of the climatological forecast $\bar{y}=\frac{1}{T} \sum y_{t}$. The values are often converted to percentages, so that $100 \%$ indicates perfection. Any score greater than $0 \%$ indicates superior performance to the climatological forecast.

Although the mean-square-error and Nash-Sutcliffe statistics are useful for quantifying the bias of forecast systems, they ignore any quantification of uncertainty. Scoring rules can be used to assess probabilistic forecasts, as they judge forecasts not only on their mean prediction, but also on the accuracy of the uncertainty quantification (see Jolliffe and Stephenson (2003) for an introduction). A score is said to be proper if it is optimized for well-calibrated probability assessments (Gneiting and Raftery, 2007), and propriety is considered an essential attribute in scientific forecast evaluation. We use the continuously ranked probability score (CRPS) (Gneiting and Raftery, 2007), which is a proper scoring rule. If $\pi(\cdot)$ is the density function of the forecast and if $y$ is the observation, then it can be shown that the CRPS can be calculated as

$$
\operatorname{crps}(\pi, \tilde{y})=\mathbb{E}_{\pi}\|Y-y\|-\frac{1}{2} \mathbb{E}_{\pi}\left\|Y-Y^{\prime}\right\|
$$


where $Y$ and $Y^{\prime}$ are independent copies of a random variable with probability density function $\pi(\cdot)$. This representation allows the CRPS to be estimated by a Monte Carlo estimate using an ensemble of forecasts. Note that if the forecast is deterministic (so that $\pi(y)$ is the Dirac delta function $\delta_{Y}(y)$ ), then Equation (10) reduces to the absolute error, $\operatorname{crps}\left(\delta_{Y}, y\right)=|Y-y|$. Hence, the CRPS generalises the absolute error, allowing us to compare probabilistic and deterministic forecasts.

We compare forecasting systems by calculating the average score across a sequence of observations,

$$
\mathrm{CRPS}=\frac{1}{T-k} \sum_{t=1}^{T-k} \operatorname{crps}\left(\pi_{t, k}, y_{t+k}\right)
$$

where $\pi_{t, k}$ is the distribution of the $k$-step-ahead forecast. Both scores are written in their negative orientation, so that the forecast system with the smallest value is preferred. We convert the raw CRPS value into a skill score by comparing it to the score attained by a reference forecast (such as climatology) in the same way the Nash-Sutcliffe statistic converts raw mean-square-error values into a percentage by comparing the forecast with climatology $\bar{y}$. We define the continuously ranked probability skill score (CRPSS) to be

$$
\text { CRPSS }=1-\frac{\text { CRPS }_{\text {forecast }}}{\text { CRPS }_{\text {reference }}},
$$

which can also be converted into a percentage. Finally, plots of the forecast errors versus the fitted values can also be used to assess the forecasting system. 


\section{Case study: Rainfall-runoff simulator}

The supplementary material contains a simulation study in which the motion of an object in freefall is simulated with no air resistance. We demonstrate that noisy observations of the object's location can be used to infer the error in the dynamics of the simulator with great accuracy. In this section we focus on a more complex simulator from hydrology that has been the subject of several previous analyses in the literature on uncertainty quantification in computer experiments (Kuczera et al., 2006; Reichert and Mieleitner, 2009; Conti et al., 2009). The logSPM simulator is a conceptual rainfall-runoff model from the saturated path modelling (SPM) family (Kavetski et al., 2003) used to model the conversion of rainfall into runoff. The model can be considered as three linked conceptual stores (representing soil, ground, and river water stores) with flow between, in, and out of the compartments at different rates. Each store can be thought of as a box, with a base area equal to the area of the catchment, containing a varying depth of water (see Figure 1). Water enters the catchment area as rain and leaves either through river discharge, evaporation, or percolation to deep aquifers. We model the system by a three dimensional temporally varying state vector, denoted $\mathbf{h}(t)=\left(h_{\text {soil }}(t), h_{\mathrm{gw}}(t), h_{\text {river }}(t)\right)$, which represents the spatially averaged depth of water in each store (measured in $\mathrm{mm}$ ) at time $t$. The mathematical specification of the simulator is given by mass balance equations for each of the three conceptual stores.

1. The depth of water in the soil store is denoted $h_{\text {soil }}(t)(\mathrm{mm})$, and increases at rate $\left(1-f_{\text {sat }}(t)\right) R(t)$, due to mass flux from rain, $R(t)$ 
(mm/day), minus surface runoff, $R(t) f_{\text {sat }}(t)$. The proportion of rain diverted to overland flow depends on the soil saturation, modelled as

$$
f_{\mathrm{sat}}(t)=\frac{1}{1+\phi_{\mathrm{F}} \exp \left(-\phi_{\mathrm{s}} h_{\mathrm{soil}}(t)\right)}-\frac{1}{\phi_{\mathrm{F}}+1} .
$$

Water in the soil store decreases due to lateral subsurface flow to the river store at rate $\phi_{\text {lat }} f_{\text {sat }}(t)$, percolation to the ground water store at rate $\phi_{\mathrm{gw}} f_{\mathrm{sat}}(t)$, and evapotranspiration at rate $f_{\mathrm{et}}(t) P(t)$, where $P(t)$ is the potential evapotranspiration ( $\mathrm{mm} /$ day), and the ratio of actual to potential evapotranspiration is related to the soil saturation by the model

$$
f_{\mathrm{et}}(t)=1-\exp \left(-\phi_{\mathrm{et}} h_{\mathrm{soil}}(t)\right)
$$

Mathematically,

$$
\frac{\mathrm{d} h_{\text {soil }}}{\mathrm{d} t}=\left(1-f_{\mathrm{sat}}(t)\right) R(t)-\phi_{\mathrm{lat}} f_{\mathrm{sat}}(t)-\phi_{\mathrm{gw}} f_{\mathrm{sat}}(t)-f_{\mathrm{et}}(t) P(t) .
$$

2. The ground water store (deep aquifers) is a linear reservoir with depth $h_{\mathrm{gw}}(t)(\mathrm{mm})$. The depth increases due to percolation from the soil at rate $\phi_{\mathrm{gw}} f_{\mathrm{sat}}(t)$, and decreases due to base flow to the river store at rate $\phi_{\mathrm{bf}} h_{\mathrm{gw}}(t)$, and percolation to deep aquifers at rate $\phi_{\mathrm{dp}} h_{\mathrm{gw}}(t)$ :

$$
\frac{\mathrm{d} h_{\mathrm{gw}}}{\mathrm{d} t}=\phi_{\mathrm{gw}} f_{\mathrm{sat}}(t)-\left(\phi_{\mathrm{bf}}+\phi_{\mathrm{dp}}\right) h_{\mathrm{gw}}(t)
$$

3. The river water store temporarily delays the water flow in the river, and is modelled as a linear reservoir of depth $h_{\text {river }}(t)(\mathrm{mm})$. The depth 
increases due to surface runoff at rate $R(t) f_{\text {sat }}(t)$, lateral subsurface flow at rate $\phi_{\text {lat }} f_{\text {sat }}(t)$, and base flow from groundwater at rate $\phi_{\mathrm{bf}} h_{\mathrm{gw}}(t)$. It decreases due to river flow out of the watershed at rate $\phi_{\mathrm{r}} h_{\text {river }}(t)$ :

$$
\frac{\mathrm{d} h_{\text {river }}}{\mathrm{d} t}=R(t) f_{\mathrm{sat}}(t)+\phi_{\mathrm{lat}} f_{\mathrm{sat}}(t)+\phi_{\mathrm{bf}} h_{\mathrm{gw}}(t)-\phi_{\mathrm{r}} h_{\text {river }}(t)
$$

The final output of the simulator is the river flow, $Q_{r}(t)$, which is the product of the watershed area $A_{w}$ and the river runoff flux $\phi_{\mathrm{r}} h_{\text {river }}(t)$ :

$$
Q_{r}(t)=A_{w} \phi_{\mathrm{r}} h_{\mathrm{river}}(t)
$$

See Figure 1 for a visual representation of the simulator. The two external forcing functions relate to weather conditions for the day in question; the rain, $R(t)$, and the potential evapotranspiration, $P(t)$. There are eight simulator parameters, denoted $\phi$., which we fixed at values estimated in Reichert and Mieleitner (2009), with $\phi_{\mathrm{s}}=0.02, \phi_{\mathrm{F}}=125, \phi_{\mathrm{et}}=0.016, \phi_{\mathrm{lat}}=1.5, \phi_{\mathrm{gw}}=$ $4.9, \phi_{\mathrm{bf}}=0.0002, \phi_{\mathrm{r}}=0.6$, and $\phi_{\mathrm{dp}}=0.02$. In a more comprehensive analysis, we may wish to let these parameters vary and estimate them along with the discrepancy function. However, for the purposes of this paper, we suppose we are given a calibrated simulator that we treat as a black-box, for which we then attempt to characterize and quantify the discrepancy.

\section{FIGURE 1 ABOUT HERE.}

Data are available from the Abercrombie watershed in New South Wales, Australia, from the year 1972 to 1976. Of the three state variables, only a function of the river flow $h_{\text {river }}(t)$ is observed, which again highlights the 
difficulty faced when quantifying model error: noisy observations of one of the three state vectors are used to estimate the uncertainty in the dynamics of all three quantities. Reichert and Mieleitner (2009) and Kuczera et al. (2006) examined the logSPM simulator for the Abercrombie watershed using the same data as we use below. Both approaches focused on allowing the simulator parameter values $(\phi$.$) to change through time: Kuczera et al. (2006)$ looked for storm dependence in the parameter values; Reichert and Mieleitner (2009) used stochastic model parameters and introducted multipliers onto the forcing terms to correct for input errors, and then inferred the implied dynamics of the parameters through time. We prefer to take a different approach and use constant (calibrated) simulator parameters, and instead look to learn a functional form for the simulator discrepancy.

Our statistical framework for relating the simulator to the observations, can be broken down into two parts. We start by relating the simulator dynamics to the system, before then describing a model relating the system to the observations. For the discrepancy model we used a linear combination of the three state variables and the two forcing functions, a constant bias term, plus white noise Gaussian residuals for each of the three dimensions in the dynamics:

$$
\boldsymbol{\delta}(\mathbf{h}, \mathbf{u})=\left(\begin{array}{c}
\delta_{s}(\mathbf{h}, \mathbf{u}) \\
\delta_{g w}(\mathbf{h}, \mathbf{u}) \\
\delta_{r}(\mathbf{h}, \mathbf{u})
\end{array}\right)+\boldsymbol{\epsilon}=\left(\begin{array}{c}
a_{s}+\mathbf{b}_{s}^{\top} \mathbf{h}+\mathbf{c}_{s}^{\top} \mathbf{u} \\
a_{g w}+\mathbf{b}_{g w}^{\top} \mathbf{h}+\mathbf{c}_{g w}^{\top} \mathbf{u} \\
a_{r}+\mathbf{b}_{r}^{\top} \mathbf{h}+\mathbf{c}_{r}^{\top} \mathbf{u}
\end{array}\right)+\boldsymbol{\epsilon}
$$


where $\mathbf{h}=\left[h_{\mathrm{s}} h_{\mathrm{gw}} h_{\text {river }}\right]^{\top} \in \mathbb{R}^{3}$ is the state vector and $\mathbf{u}=[R P]^{\top} \in \mathbb{R}^{2}$ the two weather forcing functions. The linear parameters for the soil dynamics discrepancy are grouped in the vectors $\mathbf{b}_{s}=\left[\begin{array}{lll}b_{s, 1} & b_{s, 2} & b_{s, 3}\end{array}\right]^{\top} \in \mathbb{R}^{3}$ and $\mathbf{c}_{s}=$ $\left[\begin{array}{cc}c_{s, 1} & c_{s, 2}\end{array}\right]^{\top} \in \mathbb{R}^{2}$, whilst the constant bias is given by the scalar $a_{s}$. Similarly, the other vectors $a_{g w}, a_{r}, \mathbf{b}_{g w}, \mathbf{b}_{r}, \mathbf{c}_{g w}, \mathbf{c}_{r}$ represent the same coefficients for the ground water and river state dynamics. The remaining discrepancy is modelled by Gaussian white noise, with $\boldsymbol{\epsilon} \sim \mathcal{N}(\mathbf{0}, \Sigma)$ where $\Sigma$ is a diagonal matrix with diagonal entries $\left(\sigma_{s}^{2}, \sigma_{g w}^{2}, \sigma_{r}^{2}\right)$. More complex choices, such as non-diagonal choices for $\Sigma$, heteroscedastic variances, and more complex structural forms in Equation (11) can be considered within this framework.

To relate the observations to the system, we follow Reichert and Mieleitner (2009) and apply a transformation (Box and Cox, 1964) to the observations and predicted system value in order to reduce the heteroscedasticity of the residuals. We assume independent identically distributed Gaussian measurement error on the transformed river flow, $\log \left(Q_{r}+\lambda\right)$, so that

$$
\log \left(Q_{r}+\lambda\right) \sim \mathcal{N}\left(\log \left(A_{w} \phi_{\mathrm{r}} h_{\mathrm{river}}(t)+\lambda\right), s^{2}\right)
$$

where we take the measurement variance to be $s^{2}=0.1\left(A_{w}=2770 \mathrm{~km}^{2}\right.$ for the Abercrombie catchment). The effect of applying the logarithmic transformation to the data is to induce a heteroscedastic variance on the measurement process, so that on days with small average river flows the measurements are assumed to have a smaller variance than on days for which the average river flow was large.

TABLE 1 ABOUT HERE. 
To train the discrepancy model $\boldsymbol{\delta}(\mathbf{h}, \mathbf{u})$, we used a half year period (180 days) of contiguous observations from the Abercrombie dataset (observations from 16 June 1975 till 11 December 1975). We used $N=2000$ filtering particles and $M=50$ smoothed trajectories in the MCEM algorithm described in Section 2.2. We tested various starting points for the parameters, and although some variation in the estimated values is observed due to using the Monte Carlo EM algorithm, we found that this variation did not have a large effect on the predictive power of the forecasting system. The estimated maximum-likelihood values are given in Table 1 . Notice that the estimated variance term for the river discrepancy function is several orders of magnitude smaller than for the soil or ground water discrepancy. This is expected, as we observe the river flow, but not the other two water stores. In general we find that inferring relationships involving observed quantities (rain, potential evapotranspiration, and river flow) is easier than inferring relationships involving the unobserved soil and ground water stores.

The raw parameter estimates are not particularly informative. To assess the impact of our efforts we need to examine the predictive performance of the forecasting system. We do this by reporting the mean square error (MSE), the Nash-Sutcliffe statistic (NS), and the continuously ranked probability skill score (CRPSS). We use the bootstrap particle filter (see the supplementary material) to find a weighted sample of particles $\left\{W_{t}^{(i)}, \mathbf{h}_{t}^{(i)}\right\}$ which approximates $\pi\left(\mathbf{h}_{t} \mid Q_{1: t}\right)$, and then run the system forwards in time for each particle to find the one- and five-step-ahead predictions, which can then be compared with the observations. We propagate each particle $\mathbf{h}_{t}^{(i)}$ through the system dynamics (Equation (13)) $k$ times to get a weighted sample of 
particles $\left\{W_{t}^{(i)}, \mathbf{h}_{t+k}^{(i)}\right\}$ which approximate the density $\pi\left(\mathbf{h}_{t+k} \mid Q_{1: t}\right)$. Finally, we propagate the particles through the observation process (Equation 12), adding Gaussian noise, before applying the inverse Box-Cox transformation to get values which can be directly compared with the raw observations. Let $Q_{t+k}^{r e p}$ denote the theoretical replications of the $(t+k)^{t h}$ observation, each of which will have an associated weighted $W_{i}$, giving a weighted sample of points $\left\{W_{t}^{(i)}, Q_{t+k}^{r e p,(i)}\right\}$ that approximates the predictive distribution $\pi\left(Q_{t+k}^{r e p} \mid Q_{1: t}\right)$. This distribution can then be compared to the observed value $Q_{t+k}$, taking care to use weighted averages to calculate the predictive mean and variance.

We compare the performance of three different forecasting systems:

(ODE) logSPM with measurement process only (no simulator error). A common assumption made when using complex simulators is to assume that the observations arise from the simulator prediction plus measurement error, ignoring any simulator discrepancy. We use this forecasting system as the benchmark against which we measure any improvements made by quantification of the simulator discrepancy. The observation process is applied $N$ times to get an ensemble comparable with that generated by the other forecasting systems.

(VAR) $\operatorname{logSPM}$ plus a white noise simulator discrepancy and measurement process. We assume no deterministic bias in the model discrepancy (setting $\mathbf{a} .=\mathbf{b} .=\mathbf{c} .=0$ in Equation (11)) and use system dynamics $\mathbf{h}_{t+1}=\mathbf{f}\left(\mathbf{h}_{t}, \mathbf{u}_{t}\right)+\boldsymbol{\epsilon}_{t}$ with $\quad \boldsymbol{\epsilon}_{t} \sim \mathcal{N}(0, D)$, where $D$ is a diagonal matrix. We estimated the variances to be $\sigma_{s}^{2}=97.6929, \sigma_{g w}^{2}=4.4354$ and $\sigma_{r}^{2}=0.0004$ using the MCEM algorithm. 
(FULL) logSPM plus full discrepancy model and measurement process. We assume the system dynamics are described by

$$
\mathbf{h}_{t+1}=\mathbf{f}\left(\mathbf{h}_{t}, \mathbf{u}_{t}\right)+\boldsymbol{\delta}\left(\mathbf{h}_{t}, \mathbf{u}_{t}\right)
$$

During the assessment phase, the parameter estimates for $\boldsymbol{\delta}$ remain fixed at the values shown in Table 1 .

Tables 2 and 3 show the results from assessing the three forecasting systems on the training data (data from 16 June 1975 to 11 December 1975), for the one- and five-step-ahead predictions. We can see that the system that uses the full discrepancy model (Equation (13)) out performs the other two systems on all three measures. The inclusion of any simulator discrepancy, VAR or FULL, leads to superior predictions over the simulator only system (ODE). The use of the full discrepancy model (FULL) does bring improvement over the variance only model (VAR), but not by a great amount. Figure 2 shows the fitted residuals for the ODE and FULL forecast systems. Both plots show evidence of correlated residuals, showing that further modelling improvements could still be made, although the correlation is much less extreme when using the full discrepancy. The simulator only residuals are not centred around zero showing a systematic departure from the modelling assumptions, whereas the residuals for the discrepancy model are centred around the line $y=0$, as would be expected if the model were true. Also plotted are dashed lines showing two standard deviations either side of $y=0$, at $y= \pm 2 s$ where $s$ is the standard deviation of the measurement process. If the assumed level of measurement error is accurate, then we would expect ap- 
proximately $95 \%$ of the 180 observations to lie within these two dashed lines if the simulator was perfect. This occurs for the full discrepancy forecasting system, but is clearly not the case for the simulator only system (ODE).

\section{TABLES 2 AND 3, AND FIGURE 2 ABOUT HERE.}

If we test the forecasting systems on an independent data set, i.e., on data that was not used in the training procedure, then the results are not always so positive and it is possible to make worse predictions using the full discrepancy model than when simply using the simulator only. For example, testing the forecasting systems on the same period, but from the year 1976, yields a CRPSS of $60 \%$ for the ODE system, but a value of only $21 \%$ for the FULL system (VAR scores best with $81.4 \%$ ), which is superior to climatology, but poorer than the deterministic ODE model. There are a few reasons why we believe we see this drastic drop off in performance. The first is that the results here were obtained after fitting the model to a short period of only 180 days. As found in Kuczera et al. (2006), the simulator discrepancy is largest during periods of high rainfall (storms). For the training data used there was essentially only a single large storm during this time, and so it seems likely that we have over-fit the model. By using a longer training period of data collected during more representative conditions, we hope to be able to solve the problem of overfitting. We also found evidence of seasonal dependence, with the simulator discrepancy taking a different form in summer months to that found in the winter months. We could attempt to correct this by either fitting separate discrepancy functions during the different seasons (assuming we have enough data to do this), or by including an element of seasonal dependence into the structural form of the discrepancy. 
Finally, it should be noted that the discrepancy model used is extremely simple. Extending the model to allow heteroscedastic variances in the discrepancy model (i.e., making $\operatorname{Var}(\epsilon)$ state dependent) either through the use of generalised linear models, or through another normalising transformation, may lead to an improvement in the quantification of uncertainty. The simulator discrepancy is largest during storms, and relatively small during periods of minimal rain, however the model we have fit here only allows for a single variance for the discrepancy, regardless of the weather, and so is a compromise between the two different situations. Finally, using a more complex, or non-parametric mean function (such as a Gaussian process) for the discrepancy in Equation (11) would allow us greater flexibility to capture any signal about the shape of the discrepancy function.

\section{Discussion}

If we wish to make predictions that take uncertainty into account then we

must include some description of simulator discrepancy. In this paper, we specified a statistical model for the simulator discrepancy function and have then shown how to use a training period of simulator predictions and subsequent observations to calibrate the statistical model. The focus here was on simple linear models for $\delta$ with homoscedastic error. Several immediate extensions are possible within this framework, such as the use of general linear models to allow heteroscedastic errors with state dependent variance, as well as allowing for correlation between different dimensions of the discrepancy function. We focused solely on quantifying simulator discrepancy, not on sim- 
ulator calibration. In the case where we also wished to estimate uncertain simulator parameters we could either calibrate the simulator before fitting the discrepancy model, as done in this paper, or attempt to jointly infer both sets of parameters. A joint approach is preferable, but raises computational and statistical problems and has not been considered in this paper. We suspect that in most problems a high degree of non-identifiability would exist among the simulator and discrepancy parameters.

The method proposed is computationally expensive, as it requires the repeated use of a particle filter embedded within the EM algorithm, which in turn requires repeated draws from the simulator. For expensive dynamical simulators, we could dynamically emulate the simulator as described in Conti et al. (2009), and use the emulator as a cheap statistical surrogate for the simulator to decrease computation time. To avoid running the particle filter an excessive number of times, we used a maximum likelihood approach to estimate the parameters in the discrepancy function. However, fixing the parameters at their maximum likelihood values ignores the uncertainty in the estimates. This could be avoided with a Bayesian approach, but at the expense of further computation.

Finally, note that even for simulators with a box structure (non-spatial) this is a hard problem, as typically we are trying to infer errors in the dynamics of variables that are never observed. For spatially distributed simulators (and many environmental systems originate from conservation laws in both space and time, and thus have spatial and temporal properties) the problem is harder still. Developing discrepancy models for spatially distributed simulators would either require dense (in space and time) observations, or 
strong prior knowledge of the discrepancy functional form. Where dense observations are available, for example in a heavily instrumented catchment, or measurement campaign, the approaches presented in this paper could be applied, replacing the regression functions in the discrepancy term (Equation (11)) with spatially distributed functions, such as radial basis functions, or spatial splines. This would maintain the relative simple parametric form for the discrepancy, but introduces the challenge of locating and setting the number of basis functions/knot points. Further work is needed to explore whether such methods can realistically be applied to complicated spatially distributed simulators.

\section{References}

Beven, K., 2006. A manifesto for the equifinality thesis. J. Hydrol. 320, 18-36.

Box, G. E. P., Cox, D. R., 1964. An analysis of transformations. J. Roy. Stat. Soc. B-Met. 26, 211-252.

Caffo, B. S., Jank, W., Jones, G. L., 2005. Ascent-based Monte Carlo expectation-maximization. J. Roy. Stat. Soc. B-Met. 67 (2), 235-251.

Conti, S., Gosling, J., Oakley, J., O'Hagan, A., 2009. Gaussian process emulation of dynamic computer codes. Biometrika 96 (34), 663-676.

Crucifix, M., Rougier, J., 2009. On the use of simple dynamical systems for climate predictions. Eur. Phys. J.-Spec. Top. 174, 11-31. 
Dempster, A. P., Laird, N. M., Rubin, D. B., 1977. Maximum likelihood from incomplete data via the EM algorithm. J. Roy. Stat. Soc. B-Met. 39, 1-38.

Doucet, A., de Freitas, N., Gordon, N., 2001. Sequential Monte Carlo Methods in Practice. Springer.

Gneiting, T., Raftery, A. E., 2007. Strictly proper scoring rules, prediction, and estimation. J. Am. Stat. Soc. 102 (477), 359-378.

Godsill, S., Doucet, A., West, M., 2004. Monte Carlo smoothing for nonlinear time series. J. Am. Stat. Soc. 99 (465), 156-168.

Goldstein, M., Rougier, J., 2009. Reified Bayesian modelling and inference for physical systems (with discussion). J. Stat. Plan. Infer. 139, 1221-1239.

Gordon, N. J., Salmond, D. J., Smith, A. F. M., 1993. Novel approach to nonlinear/non-Gaussian Bayesian state estimation. IEE Proc-F 140, 107113.

Griffith, A. K., Nichols, N. K., 2000. Adjoint techniques in data assimilation for estimating model error. J. Flow Turb. Comb. 65, 469-488.

Higdon, D., Gattiker, J., Williams, B., Rightley, M., 2008. Computer model calibration using high-dimensional output. J. Am. Stat. Soc. 103, 570-583.

House, L., Goldstein, M., Rougier, J., 2011. Assessing model discrepancy using a multi-model ensemble. In submission.

Jolliffe, I. T., Stephenson, D. B., 2003. Forecast Verification: A Practitioner's Guide in Atmospheric Science. Wiley and Sons, Chichester. 
Kavetski, D., Kuczera, G., Franks, S. W., 2003. Semi-distributed hydrological modelling: a 'saturation path' perspective on TOPMODEL and VIC. Water Resour. Res. 39, 1246-1253.

Kennedy, M. C., O’Hagan, A., 2001. A Bayesian calibration of computer models (with discussion). J. Roy. Stat. Soc. B-Met. 63, 425-464.

Kuczera, G., Kavetski, D., Franks, S., Thyer, M., 2006. Towards a Bayesian total error analysis of conceptual rainfall-runoff models: Characterising model error using storm-dependent parameters. J. Hydrol. 331, 161-177.

Meng, X. L., Rubin, D. B., 1991. Using EM to obtain asymptotic variancecovariance matrices: the SEM algorithm. J. Am. Stat. Soc. 86, 899-909.

Nash, J. E., Sutcliffe, J. V., 1970. River flow forecasting through conceptual models part I - a discussion of principles. J. Hydrol. 10, 282-290.

Oakley, J. E., O'Hagan, A., 2002. Bayesian inference for the uncertainty distribution of computer model outputs. Biometrika 89, 769-784.

Oberkampf, W. L., Trucano, T. G., 2008. Verification and validation benchmarks. Nucl. Eng. Des. 238, 716-743.

Reichert, P., Mieleitner, J., 2009. Analyzing input and structural uncertainty of nonlinear dynamic models with stochastic time-dependent parameters. Water Resources Research 45, 1-19.

Saltelli, A., Chan, K., Scott, M. (Eds.), 2000. Sensitivity Analysis. Wiley, New York, USA. 
Shmueli, G., 2011. To explain or to predict? Statistical Science.

Smith, R., Tebaldi, C., Nychka, D., Mearns, L., 2009. Bayesian modeling of uncertainty in ensembles of climate models. J. Am. Stat. Soc. 104, 97-116.

Strong, M., Oakley, J. E., Chilcott, J., 2011. Managing structural uncertainty in health economic decision models: a discrepancy approach. J. Roy. Stat. Soc. C-App., in press.

Vernon, I. R., Goldstein, M., Bower, R. G., 2010. Galaxy formation: a Bayesian uncertainty analysis. Bayesian Analysis 5, 619-670.

Wei, G. C. G., Tanner, M. A., 1990. A Monte Carlo implementation of the EM algorithm and the poor man's data augmentation algorithms. J. Am. Stat. Soc. 85 (411), 699-704. 


\begin{tabular}{|l|c|ccc|cc|c|}
\hline Dimension & Bias & \multicolumn{3}{|c|}{ Linear parameters } & \multicolumn{2}{|c|}{ Forcing coeffs. } & Variance \\
& $a$. & $b_{\cdot, 1}$ & $b_{\cdot, 2}$ & $b_{\cdot, 3}$ & $c_{\cdot, 1}$ & $c_{\cdot, 2}$ & $\sigma_{.}^{2}$ \\
\hline Soil $\delta_{s}$ & 12.7803 & -0.0662 & 0.0740 & 0.8091 & -0.6254 & -2.0863 & 29.7519 \\
Ground water $\delta_{g w}$ & 6.7218 & -0.0205 & 0.0362 & -0.8516 & -0.0766 & -1.5297 & 2.7294 \\
River $\delta_{r}$ & -0.2111 & 0.0022 & -0.0019 & -0.0487 & 0.0034 & 0.0384 & 0.0005 \\
\hline
\end{tabular}

Table 1: Estimated maximum likelihood parameters for the discrepancy function described by Equation (11). Each row describes the parameter values for the discrepancy function in the dynamics of one of the three state variables representing the three conceptual water stores in the logSPM simulator.

\begin{tabular}{|c|c|c|c|}
\hline \multicolumn{4}{|c|}{ One step ahead predictions $(k=1)$} \\
\hline & MSE & NS $(\%)$ & CRPSS $(\%)$ \\
\hline ODE & 0.2764 & 74.6 & 73.2 \\
VAR & 0.1547 & 85.8 & 81.6 \\
FULL & 0.0988 & 90.9 & 85.0 \\
\hline
\end{tabular}

Table 2: Validation results for the one-step-ahead forecasts for the three forecasting systems described in the text. ODE is the deterministic logSPM simulator, VAR is the simulator plus a white noise discrepancy, and FULL is the simulator plus the estimated discrepancy function. The three measures used are the mean square error (MSE), the Nash-Sutcliffe statistic (NS), and the continuously ranked probability skill score (CRPSS). The data used in the validation was a 180 day period (16 June 1975 till the 11 December 1975). The reference forecast used for the NS statistic and the CRPSS was a Gaussian distribution with mean and variance estimated from the observations (i.e., the climatological forecast).

\section{Tables}

\section{Figures}




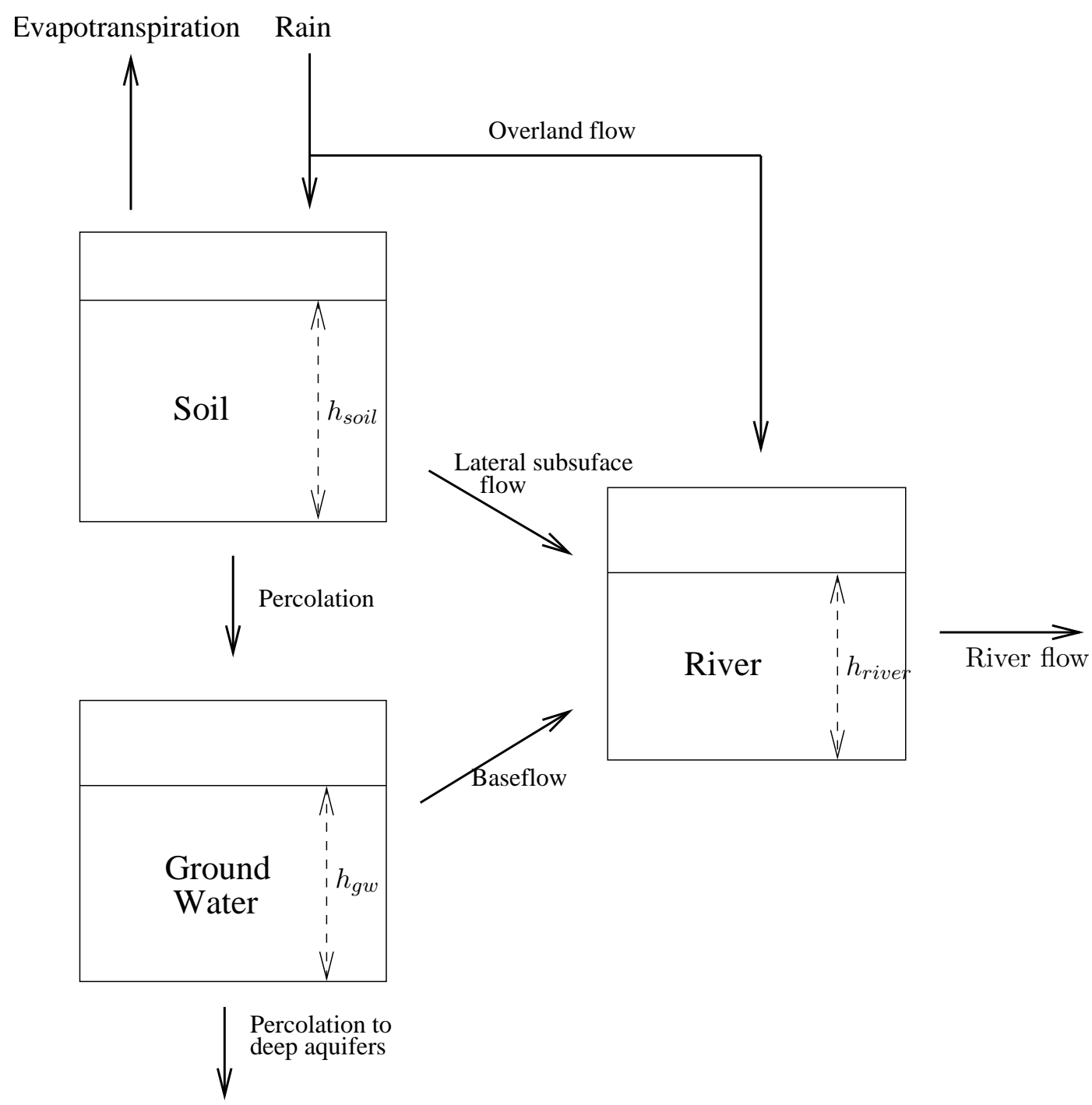

Figure 1: A visual representation of the logSPM simulator. 


\begin{tabular}{|c|c|c|c|}
\hline \multicolumn{4}{|c|}{ Five step ahead predictions $(k=5)$} \\
\hline & MSE & NS (\%) & CRPSS (\%) \\
\hline ODE & 0.2764 & 74.6 & 73.2 \\
VAR & 0.1944 & 81.0 & 79.5 \\
FULL & 0.1035 & 89.9 & 84.5 \\
\hline
\end{tabular}

Table 3: Validation results for the five-step-ahead forecasts for the three forecasting systems described in the text. The scores for the ODE system are the same as in Table 2.
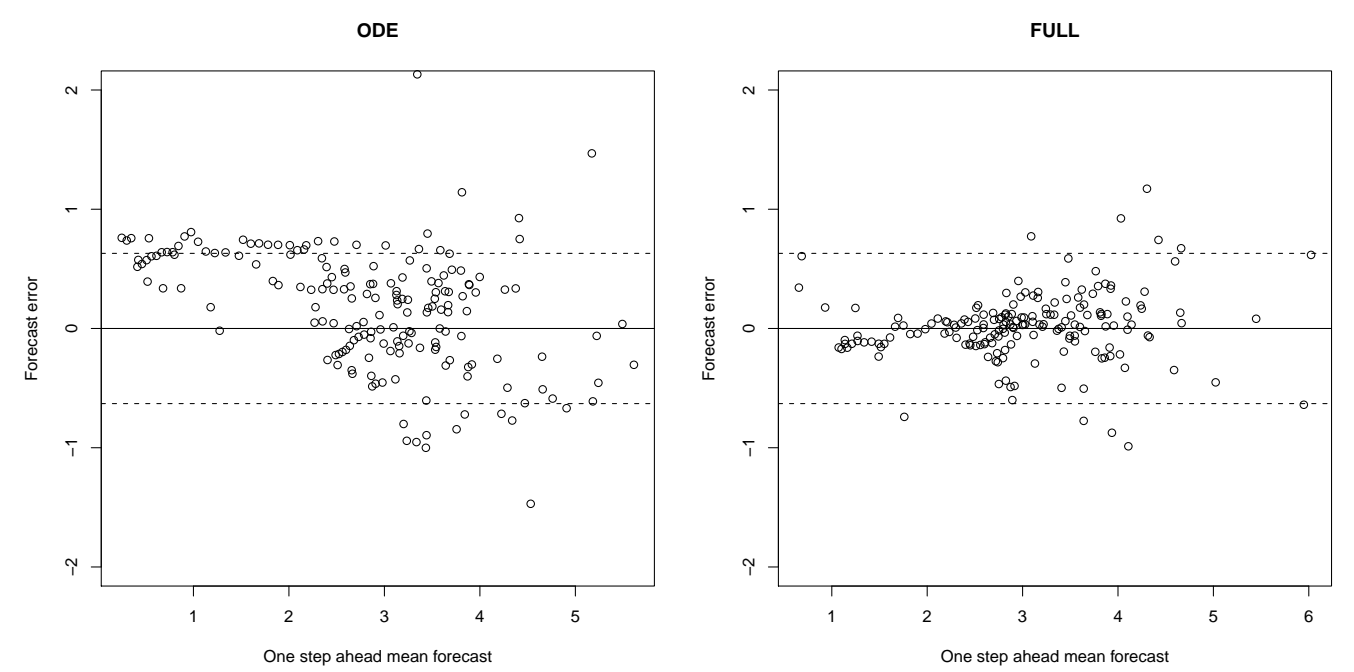

Figure 2: A residual plot showing the one-step-ahead transformed forecast errors, $\log \left(Q_{t+1}+\lambda\right)-\tilde{m}_{t}(1)$, versus the fitted values, $\tilde{m}_{t}(1)=$ $\left.\log \left(A_{w} k_{r} h_{\mathrm{river}, t+k}+\lambda\right)\right)$. The plot on the left is for the ODE forecasting system with no simulator discrepancy term, and the plot on the right is for the full discrepancy model. A forecasting system which had no simulator discrepancy would have a residual plot that looked like an uncorrelated band of residuals distributed about the line $y=0$. The dashed lines are two standard deviations (of measurement error) either side of $y=0$, giving bounds within which we would expect to see approximately $95 \%$ of the 180 points if the simulator were perfect. 